\title{
Reconstruction and Identification of Tau Leptons in ATLAS
}

\author{
Christian Limbach, on behalf of the ATLAS collaboration
}

University of Bonn,limbach@physik.uni-bonn.de

\begin{abstract}
These proceedings describe the reconstruction and identification of tau leptons in the ATLAS experiment at the LHC. Tau leptons play an important role in the measurement of Standard Model processes, in the search for new physics (Supersymmetry, heavy resonances) and in the measurement of Higgs boson properties. Hadronic tau lepton decays are reconstructed calorimeter based and separation against QCD-induced jets is done relying on shapes of energy depositions in the calorimeter as well as tracking based variables. The energy scale of tau leptons is obtained by scaling the measured momentum to the simulated one and is cross-checked in data. The tau trigger makes use of the collimated nature of tau decays and relies on a combination of objects (e.g. tau + muon) to reduce the rates and thresholds. An outlook to the improved tau reconstruction to be used in the next data-taking period of ATLAS concludes the proceedings.
\end{abstract}

\section{(C) 2011 Published by Elsevier Ltd.}

Keywords:

LHC, ATLAS, Tau, Lepton, Reconstruction, Identification, Tau Energy Scale, Trigger, Energy flow

\section{Introduction}

Tau leptons play an important role in the physics programme of the ATLAS experiment [1] at the LHC [2]. Measurements of Standard Model processes $(Z \rightarrow \tau \tau$ cross-section [3]), evidence of $H \rightarrow \tau \tau$ [4] and search for supersymmetry [5] are just a few examples. The tau lepton decays hadronically $(\approx 65 \%)$ and leptonically $(\approx 35 \%)$. Only hadronic decays are considered in the tau reconstruction. Hadronic tau decays are classified into 1prong and 3-prong decays, which contain one and three charged particles, respectively. The charged particles are mostly $\pi^{ \pm}$and can be accompanied by neutral particles, also mostly pions, $\pi^{0} 1$. Every tau decay contains a tau neutrino, so that only the visible tau energy can be measured and the true energy is not accessible. All decay products are collimated, due to the boost of the tau lepton.

\footnotetext{
${ }^{1}$ There are also decays involving charged and neutral Kaon's, but those are suppressed.
}

The main background to tau leptons are QCD induced jets. However in contrast to hadronic tau decays, their particle multiplicity increases with the energy of the jet and they are typically wider.

An illustrative comparison of a hadronic tau decay and a QCD induced jet is shown in Fig. 1

\section{Reconstruction and Identification of Tau Leptons}

This section gives a very brief summary of the in-depth description found in [7].

The reconstruction of hadronic tau decays is seeded by reconstructed and calibrated Anti-Kt jets (with distance parameter of 0.4 ) with a transverse momentum of $p_{T \text {, Calibrated }}>10 \mathrm{GeV}$. As a first step, the barycentre of the clusters ${ }^{2}$ is calculated and all clusters within a cone of size $\Delta R=0.2$

\footnotetext{
${ }^{2} \mathrm{~A}$ jet consists of several clusters, and each cluster is a set of calorimeter cells.
} 


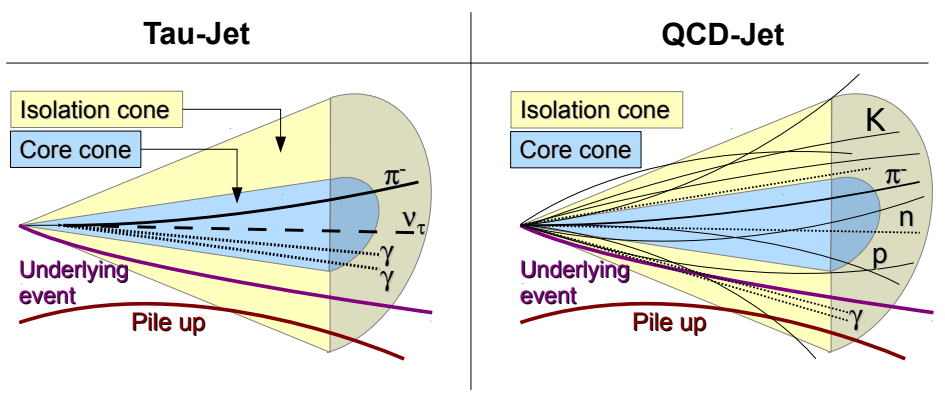

Fig. 1. Illustration of a hadronically decaying tau lepton and a QCD induced jet. The tau decay products are boosted, so that they are confined in a core cone, leaving the isolation cone rather empty.

are collected ${ }^{3}$. The position of the selected clusters is calculated with respect to the vertex from which the tau originated. The corrected positions are then used to calculate the tau axis. Another cone of $\Delta R=0.2$ is constructed around this tau axis and tracks within it are associated to the tau decay. An isolation cone with $\Delta R=0.4$ is used to collect tracks with $0.2<\Delta R<0.4$, which help to evaluate the isolation of the tau candidate (c.f. Fig. 1).

The identification of tau leptons is performed dependent on the number of prongs (i.e. tracks). It is distinguished between 1-prong and multi-prong (two or more prongs). In each category, there are several variables which separate tau leptons from QCD induced jets, an example for a variable in the 1-prong category is shown in Fig. 2. For each category, the separating variables are combined into a Boosted Decision Tree (BDT) ${ }^{4}$. Signal taus are reconstructed true taus with $|\eta|<2.3$ and $p_{T}>15 \mathrm{GeV}$ that are obtained from simulation. The background is taken from early 2012 data and consists of tau candidates within $|\eta|<2.3$ and $p_{T}>15 \mathrm{GeV}$.

Fig. 2 also shows the BDT score and its composition of tau leptons as measured in a $Z \rightarrow \tau \tau$ tag-and-probe analysis ${ }^{5}$. Based on the BDT score distribution, three working points for the identification are defined, each giving a fixed signal efficiency (for 1-prong (3-prong) taus, 70\% (65\%),

\footnotetext{
${ }^{3} \Delta R=\sqrt{\Delta \phi^{2}+\Delta \eta^{2}} . \quad \eta$ is the pseudorapidity, $\eta=$ $-\ln \tan (\theta / 2)$. $\theta$ and $\phi$ are the polar and azimuthal angles of a spherical coordinate system centred in the collision point in ATLAS, with the $x$-axis pointing towards the centre of the LHC, the $y$-axis pointing to the earth surface and the $z$-axis pointing along the beam direction.

${ }^{4}$ Boosted Decision Tree, a multivariate method exploiting correlations between variables to increase separation power.

${ }^{5}$ Tag-and-probe means selecting a light lepton from one tau decay, the tag, and then using the hadronic decay of the other tau as the probe for identification.
}

$60 \%(55 \%), 40 \%(35 \%))$. The signal efficiencies have been checked to be flat with respect to the true visible tau momentum and the number of primary vertices in the event.

In addition to separation against jets, the identification also separates hadronic taus from electrons (by using a dedicated BDT) and from muons (by vetoing tau candidates (a) in analyses that overlap with muons and (b) with very high or low electromagnetic fractions) [7].

\section{Tau Energy Scale}

The tau energy scale (TES) is a correction factor to scale the reconstructed, visible tau momentum, $p_{\tau, \text { reco, vis }}$ back to the true visible tau momentum

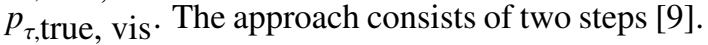

In a first step, the momentum is corrected for pile-up effects. This is done by evaluating the energy contribution from pile-up per vertex in different bins of pseudorapidity $\eta$. Thus, given the number of vertices in the event, the pile-up contribution can be calculated and subtracted from the measured momentum.

The second step is to scale this pile-up-corrected momentum to the true visible momentum by using response curves. The response curves are a function of the reconstructed tau momentum, binned in pseudorapidity $\eta$ and the number of prongs, and give the ratio between the reconstructed and true visible tau momentum. Fig. 3 shows the response curve for 1-prong taus for $|\eta|<0.3$. Thus, by dividing the pile-up-corrected momentum by the response, the reconstructed momentum is scaled to the true visible one.

The total uncertainty of the TES is typically below $4 \%$. Uncertainties that have been taken into account are uncertainties from single particle responses, underlying event model, different pile-up 

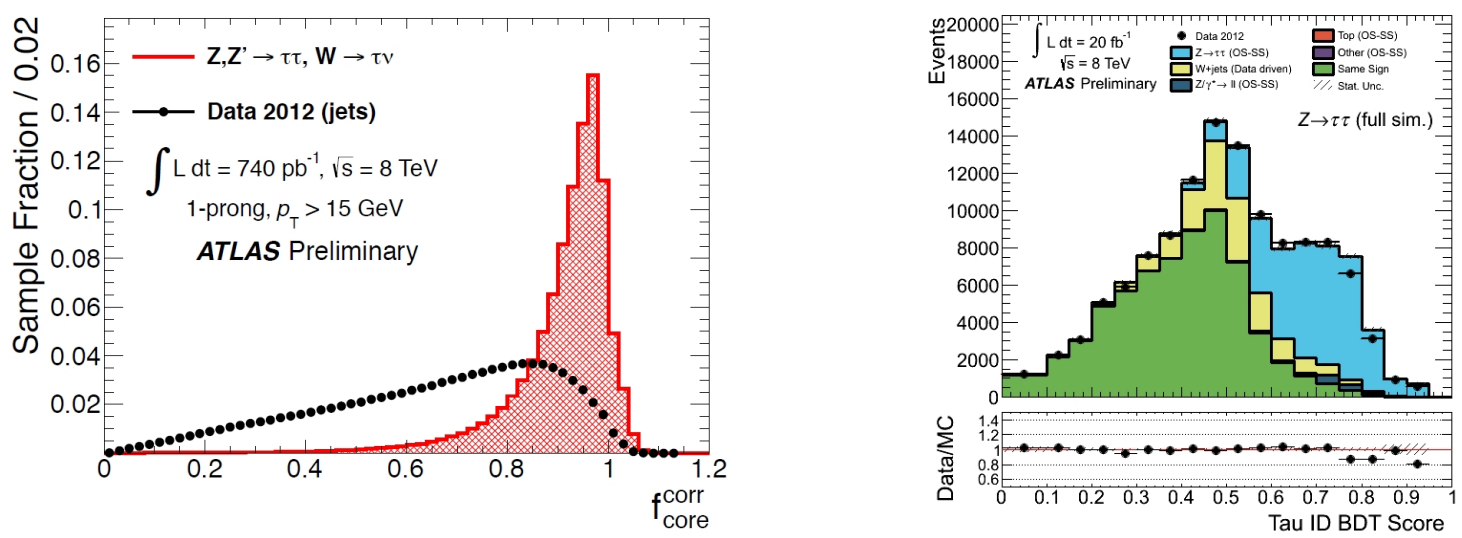

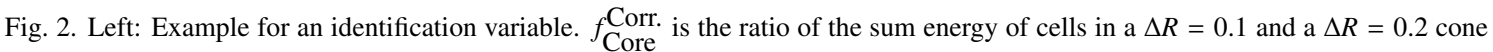
around the tau axis. Because taus are collimated, the distribution peaks around one for simulated tau decays. [7]

Right: BDT score of tau candidates as obtained from a $Z \rightarrow \tau \tau$ tag-and-probe analysis. Tau candidates from processes involving real taus gather at higher BDT scores than candidates from processes without real taus. [8]

conditions and the amount of dead material.

The TES has been measured in data (right plot of Fig. 3). The TES that is used in the simulation has been varied in template fits in order to find the value giving the best agreement between data and simulation. Results obtained here are consistent with the expectations from simulations described above.

\section{Trigger}

This section briefly summarises the tau triggers as described in [10]. The trigger system in the ATLAS detector has three levels.

The first level relies on so-called trigger towers, which are the sums of calorimeter cells across layers, such that their size is about $\Delta \eta \times \Delta \phi=0.1 \times 0.1$. This combination is done for the electromagnetic and hadronic calorimeter separately. Energy in the core region of these trigger towers is expected to be small for taus, because of the collimation of the decay products.

The second level of the trigger has access to the full granularity of the calorimeter and to a simplified and faster version of the tracking. Thus, variables describing the shape of the energy depositions can help to distinguish taus from QCD induced jets.

Finally, the event filter can rely on the complete detector information, and employs a BDT which is similar to the one used in the offline reconstruction.

The left part of Fig. 4 shows the rates of several tau triggers, which are often a combination of objects to keep the energy thresholds small.

The trigger efficiency has been measured in data using a $Z \rightarrow \tau \tau$ tag-and-probe analysis, and the trigger efficiency with respect to the offline reconstruction efficiency as a function of the offline tau $p_{T}$ is shown in the right part of Fig. 4. Good agreement between data and simulation is observed, as well as a flat trigger efficiency above $35 \mathrm{GeV}$ offline tau $p_{T}$. The trigger efficiency as a function of pile-up has also been studied and is found to be stable within 5-10\%.

\section{Prospects for 2015 and beyond}

The tau reconstruction in the course of the next data-taking period will be enhanced by an energy flow approach. In this approach, the energy depositions from charged particles in the electromagnetic (EM) calorimeter are estimated and removed, so that in an ideal case only energy depositions from neutral particles remain in the EM calorimeter. These are passed into a $\pi^{0}$ identification and are used in kinematic tau variables to determine the decay mode. In addition, a significant improvement in the spatial and energy resolution is expected.

\section{Summary}

In ATLAS, tau leptons are reconstructed as a set of clusters and tracks. Their properties are exploited at trigger level and in the offline reconstruction to achieve a good separation against the main 

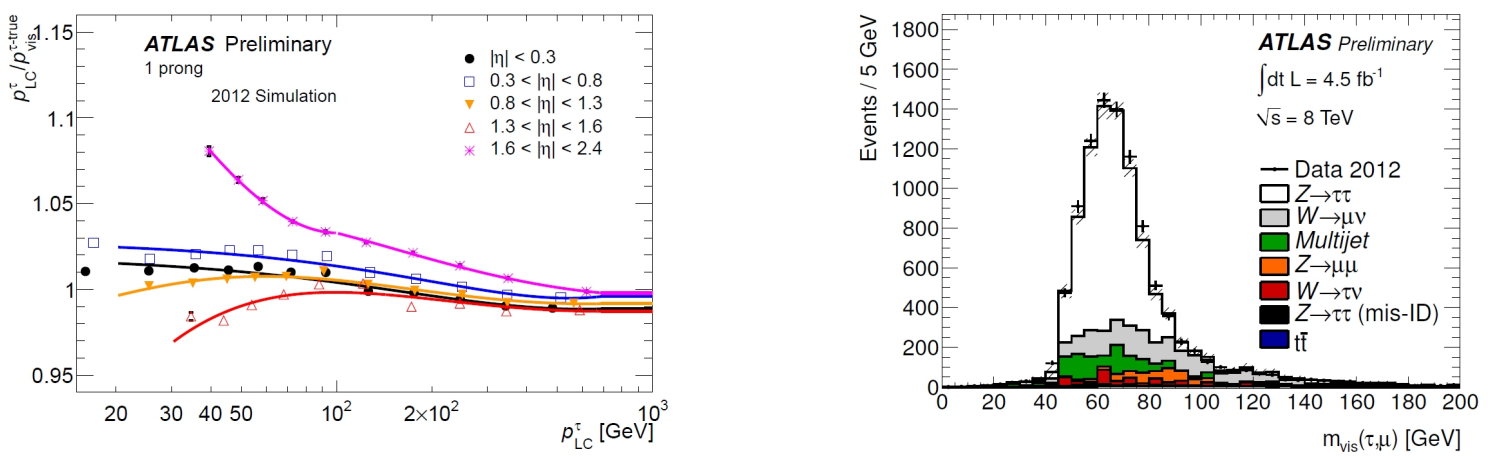

Fig. 3. Left: Response curves as a function of reconstructed $p_{T}$ for different $\eta$-bins. The pile-up-corrected tau $p_{T}$ is divided by the response to restore the scale of the true visible tau. [9]

Right: Invariant di-tau mass as obtained from a $Z \rightarrow \tau \tau$ tag-and-probe analysis. The simulated processes are scaled with different tau energy scales using template fits to find the value giving the best agreement between data and simulation. The result is consistent with the TES obtained from simulations. [9]
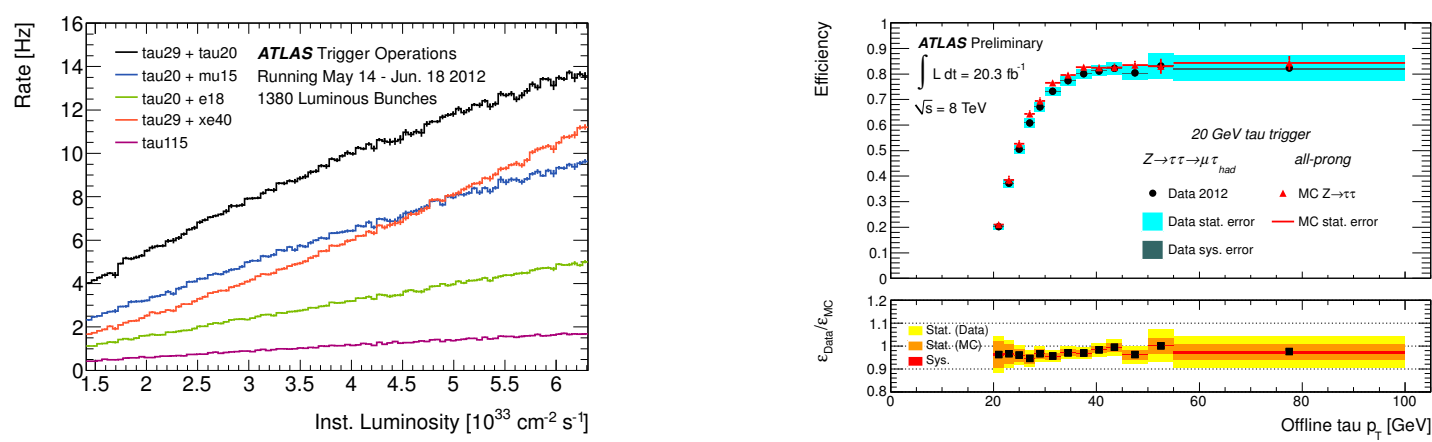

Fig. 4. Left: Trigger rate of different tau triggers as a function of the instantaneous luminosity. Tau triggers with a low threshold are possible because of their combination with other objects, either another tau (tau29+ tau20) or another object, muon, electron, or missing energy.[11]

Right: Measurement of the trigger turn-on curve. The trigger efficiency for a tau trigger with an $E_{T}$ threshold of $20 \mathrm{GeV}$ is plotted as a function of the offline tau $p_{T}$. The plateau of $80 \%$ is reached around $p_{T \text {, offline }}=35 \mathrm{GeV}$. Data and simulation agree within uncertainties. The efficiency is measured with respect to the offline signal efficiency.[11]

background, QCD induced jets, as well as electrons and muons. The TES is tuned specifically to tau decays and has an uncertainty of typically $4 \%$. Results obtained in simulations are cross-checked in data by using Standard Model processes. In the course of the next data-taking period, an energy flow based approach will enhance the tau reconstruction.

\section{References}

[1] ATLAS Collaboration, JINST 32008 S08003

[2] L. Evans and P. Bryant (editors) JINST 32008 S08001

[3] ATLAS Collaboration, ATLAS-CONF-2012-006, https://cds.cern.ch/record/1426991

[4] ATLAS Collaboration, ATLAS-CONF-2013-108, https://cds.cern.ch/record/1632191
[5] ATLAS Collaboration, CERN-PH-EP-2014-144, https://cds.cern.ch/record/1728108

[6] ATLAS Collaboration, ATL-COM-PHYS-2014-1118, https://indico.cern.ch/event/300387/session/21/contribution/55

[7] ATLAS Collaboration, ATLAS-CONF-2013-064, https://cds.cern.ch/record/1562839

[8] ATLAS Collaboration, ATL-COM-PHYS-2014-153, http://atlas.web.cern.ch/Atlas/ GROUPS/PHYSICS/TAUCP/PublicPlots/ATL-COMPHYS-2014-153/

[9] ATLAS Collaboration, ATLAS-CONF-2013-044, https://cds.cern.ch/record/1544036

[10] ATLAS Collaboration, ATLAS-CONF-2013-006, http://cds.cern.ch/record/1510157

[11] ATLAS Collaboration, https://twiki.cern.ch/twiki/bin/view/ AtlasPublic/TauTriggerPublicResults 